\title{
Computed Tomography Diagnosis of Acute Appendicitis_-Pictorial Essay
}

\author{
Aarthi Govindarajan, Bhawna Dev, Roy Santosham, Santhosh Joseph \\ Department of Radiology and Imaging Sciences, Sri Ramachandra Medical College and Research Institute, \\ Sri Ramachandra University, Chennai, India \\ E-mail:aarthi_govind@yahoo.com \\ Received October 11, 2010; revised December 25, 2011; accepted April 11, 2011
}

\begin{abstract}
Acute appendicitis is a common surgical emergency with varied clinical presentations. Early diagnosis is absolutely necessary to minimize morbidity whereas delayed or missed diagnosis can cause adverse consequences. Computed tomography is a highly accurate imaging technique for diagnosing appendicitis. Hence it plays a valuable role in selected patients with suspected appendicitis; [1]. In this essay, we review the normal Computed tomography anatomy of the appendix and the right lower quadrant and illustrate the Computed tomography signs of appendicitis and important differential diagnostic entities. The Computed tomography appearance of complications of acute appendicitis is also presented, as are issues concerning clinical presentation and duration of the symptoms. Computed tomography signs can be varied and overlooked as they say what is easy to see is also easy to miss.
\end{abstract}

Keywords: Acute Appendicitis, Multislice Computed Tomography

\section{Introduction}

Acute appendicitis is the commonest cause of acute abdomen pain which needs surgical intervention. Atypical symptoms, varied presentations and multiple differentials of abdomen pain make accurate diagnosis of appendicitis difficult clinically.

Non enhanced \& contrast enhanced Computed tomography images are helpful in noninvasive evaluation of appendicitis. The Computed tomography diagnosis of acute appendicitis has high positive and negative predictive values, $96 \%$ and $95 \%$ [2], respectively. It is also possible to reconstruct the entire form and position of appendices from successive Computed Tomography findings with high-resolution thin-slice Multidetector computed tomography images [3].

\section{Normal Anatomy}

Appendix is a narrow worm shaped blind ending tube arising from the caecum. Appendix is highly mobile and variable in length by up to $20 \mathrm{~cm}$ [4]. It can be retrocaecal, subcaecal, pelvic, preileal and post ileal in location. Most common is the retrocaecal position [5]. Appendix has a triangular mesentery, the meso appendix. Appendix is supplied by appendicular artery, a branch of ileocolic artery and venous drainage is through ileocolic vein into the superior mesenteric vein.

\section{Cases with Detailed Description}

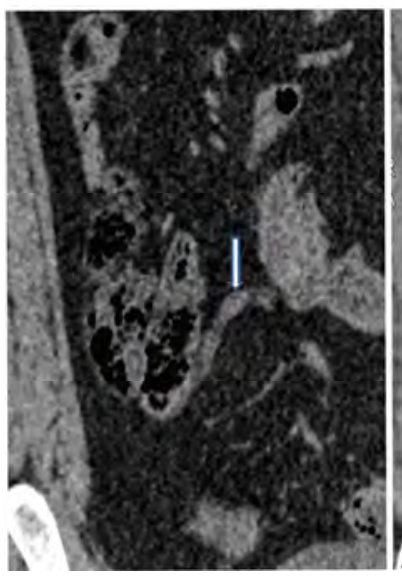

(a)

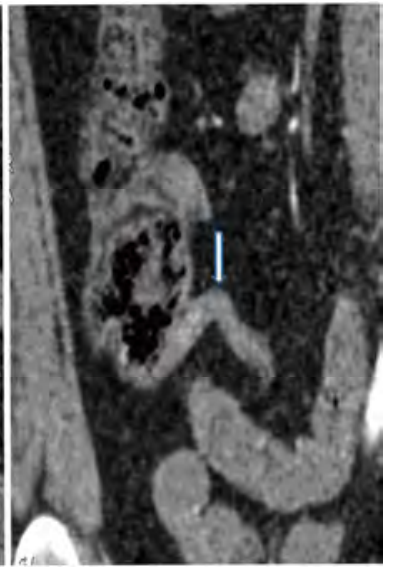

(b)
Figure 1. Non enhanced (a) and Enhanced (b) shows normal blind ending appendix arising from the caecum. 


\subsection{Case I Computed Tomography Appearance of Normal Appendix}

Patient came for evaluation of renal calculi and Computed tomographic images showed normal appendix as smooth thin walled tubular organ (arrow) surrounded by mesenteric fat (Figures 1(a) and 1(b)). Transverse diameter of normal appendix should not exceed $6 \mathrm{~mm}$ without intraluminal material, but some authors considered an upper limit of $10 \mathrm{~mm}$ with intraluminal content [6].

\subsection{Case II Acute Appendicitis}

Patient presented to Emergency Room with periumblical pain and vomiting for 2 days and Computed Tomographic images showed inflammed appendix with periappendicular mesenteric stranding (Figures 2(a), 2(b), 2(c) and 2(d)).

\subsection{Case III Retrocaecal Appendicitis with Lateral Conal Fascia Thickening and Retroperitoneal Abscess}

Patient was referred from Emergency Room to rule out acute appendicitis and Computed Tomography showed retrocaecal long inflammed appendix with adjacent retroperitoneal abscess (Figures 3(a), 3(b), 3(c), 3(d), 3(e) and 3(f))

\subsection{Case IV Long inflammed Appendix with Midline Extension}

Patient with right iliac fossa pain and fever for 3 days.

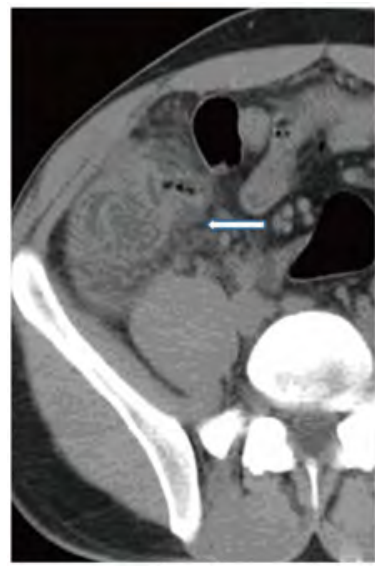

(a)

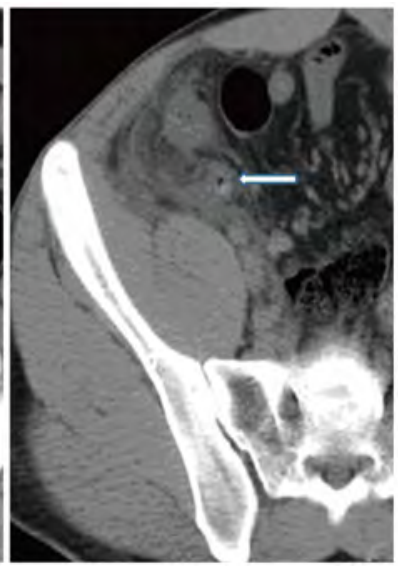

(b)

Computed Tomographic images showed long appendix crossing the midline with features of acute appendicitis (Figures 4(a), 4(b) and 4(c)).

\subsection{Case V Appendicolith without Inflammation}

Patient presented with right iliac fossa pain for 2 days and Computed Tomographic showed appendicolith with no evidence of appendix enlargement/periappendicular stranding (Figures 5(a) and 5(b))

\subsection{Case VI Appendicolith with Inflammation}

Patient complained of lower abdomen pain with vomiting. Computed Tomography showed enlarged thickened appendix with minimal periappendicular mesenteric stranding seen. (Figures 6(a) and 6(b)).

\subsection{Case VII Base Appendicitis}

Patient presented with lower abdomen pain and Computed Tomography showed acute inflammation at the take off of appendix from caecum with minimal stranding (Figures 7(a) and 7(b)).

\subsection{Case VIII Terminal Appendicitis with Perforation and Pneumoperitoneum}

Patient presented with dull aching right iliac fossa pain and Computed Tomographic images showed thickened tip of appendix with mesenteric stranding (Figures 8(a), 8(b) and 8(c)).

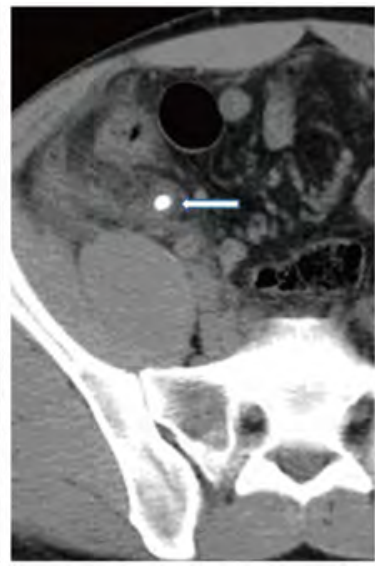

(c)

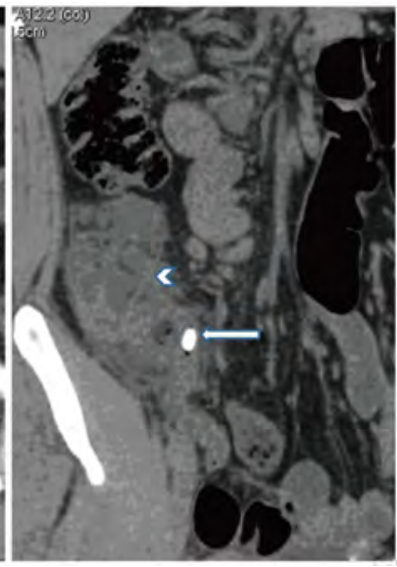

(d)

Figure 2. (a) Non-enhanced axial section of computed tomography shows diffuse thickening of distal caecum with surrounding inflammatory mesenteric stranding (arrow); (b) Non enhanced Computed Tomography shows enlarged and thickened appendix (arrow) with luminal diameter $11 \mathrm{~mm}$ in its cross section. (c) Non enhanced axial Computed Tomography shows appendicolith (arrow) measuring 9 mm within the lumen. (d) Coronal section of Non Contrast Enhanced Computed Tomography abdomen shows pericolic mesenteric fat stranding with thickening of distal ileal loops and caecum (arrow head) and appendicolith within the lumen (arrow). 


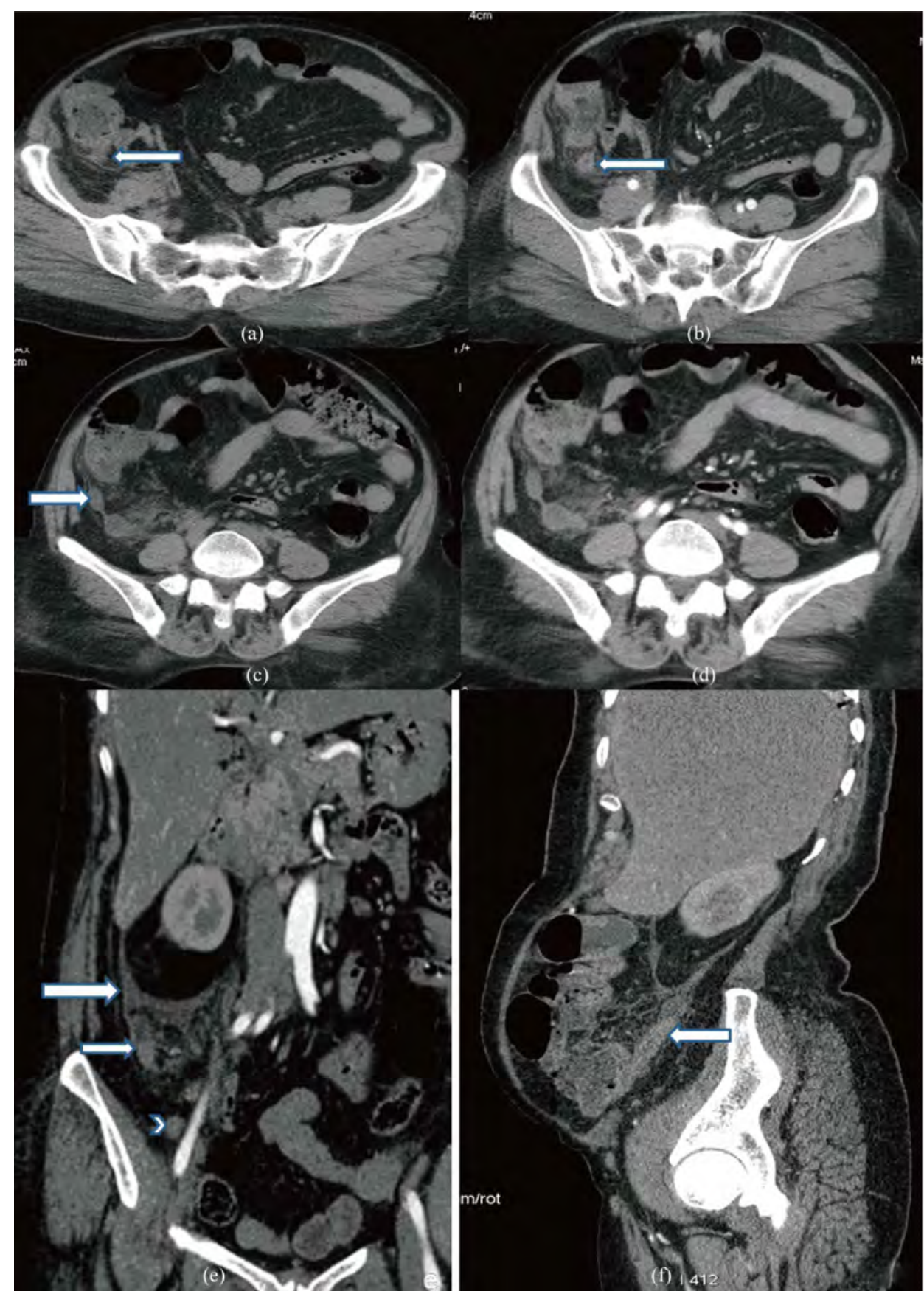

Figure 3. (a) Non enhanced axial Computed Tomography shows periappendicular stranding (arrow) at the level of take off of appendix from caecum. (b) Non enhanced axial Computed Tomography shows thickened appendix with transverse diameter of $1.3 \mathrm{cms}$ (arrow). (c) \& (d) Non enhanced (c)and enhanced (d) axial Computed Tomography shows small loculated abscess (c, arrow) in retro peritoneum adjacent to thickened appendix showing enhancement after contrast administration (d, arrow). (e) Thickened retrocaecal appendix (small arrow) leading to thickened right lateral conal fascia (arrow) with multiple mesenteric lymph nodes (arrow head). (f) Contrast Enhanced Computed Tomography oblique images shows long retrocaecal thickened appendix (arrow) $(1.3 \mathrm{~cm})$ extending up to posterior renal fascia.

\subsection{Case IX Appendicular Mass}

Right Iliac Fossa pain for 2 weeks and Computed Tomographic images showed heterogeneous mass in right iliac fossa with thickening of ascending colon and distal il- eum (Figures 9(a), 9(b) and 9(c)).

\subsection{Case X Appendicular Abscess}

Lower abdomen pain for 2 weeks and Computed Tomo 


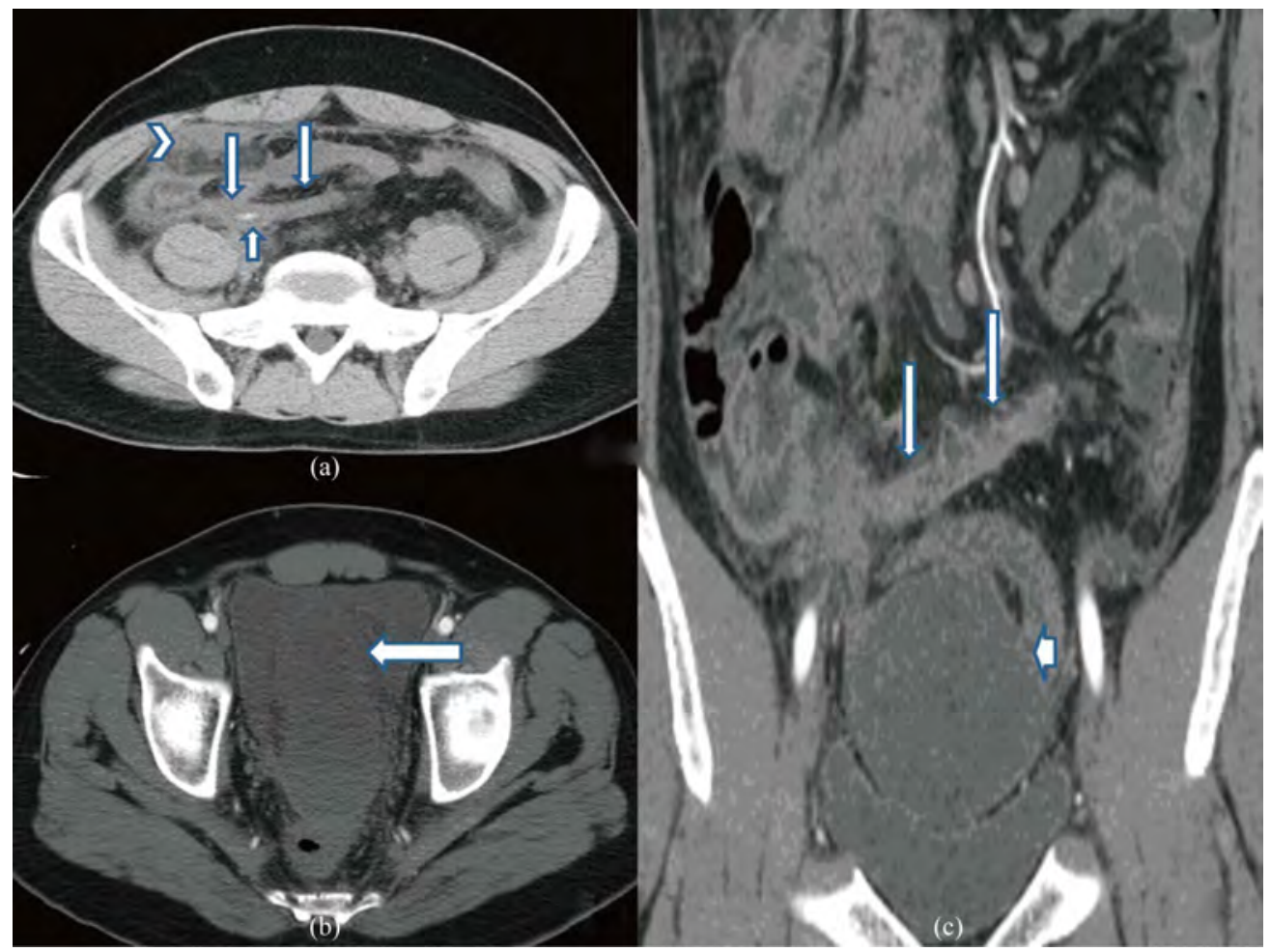

Figure 4. (a) Nonenhanced Computed Tomography shows $10 \mathrm{~cm}$ long edematous appendix (arrows) crossing the midline with luminal diameter $1.2 \mathrm{~cm}$, with surrounding fat stranding. Linear appendicolith (Small arrow) is seen within the lumen. Small pericolic fluid collection (arrowhead) is seen anterior to caecum. (b) Enhanced axial Computed Tomography shows Large fluid collection (Arrow) anterior to rectum with attenuation similar to bladder. (c) Enhanced coronal Computed Tomography shows long inflammed appendix (arrows) with periappendicular stranding. Fluid collection (arrow head) seen in pelvis.

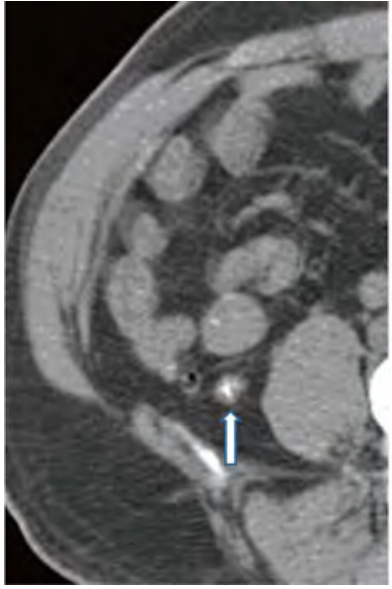

(a)

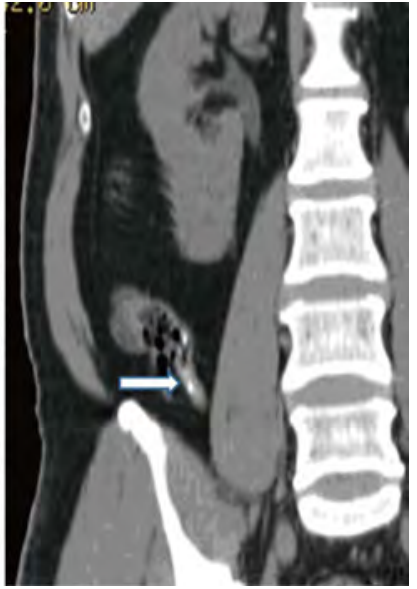

(b)

Figure 5. (a) Non enhanced axial Computed tomography shows appendicolith (arrow) noted within appendix lumen. (b) Coronal oblique images of Computed Tomography abdomen shows noninflammed appendix (arrow) with faecolith.

graphy showed irregular thick walled abscess with appendix not separately visualized (Figures 10(a) and 10(b)).

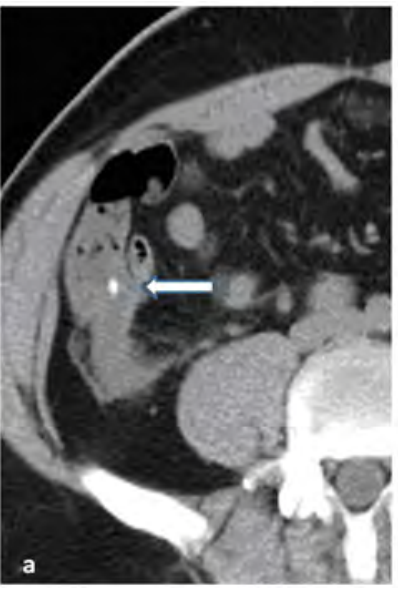

(a)

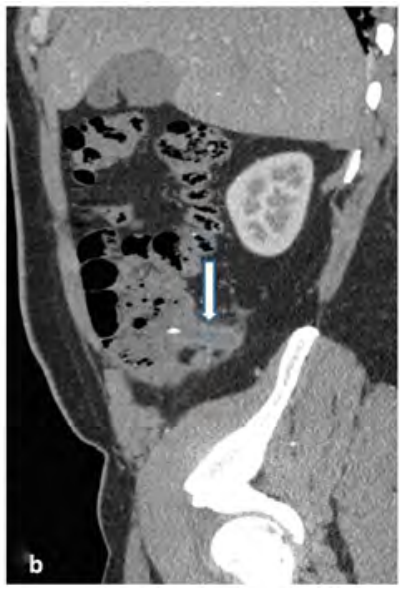

(b)
Figure 6. (a) Non enhanced axial Computed Tomography shows fluid filled thickened appendix with appendicolith (arrow) at the neck of appendix. (b) Saggital oblique nonenhanced images shows fluid filled appendix with appendicolith.

\subsection{Case XI Acute Appendicitis with Contained Perforation}

Patient complained of acute abdomen pain for 2 days 


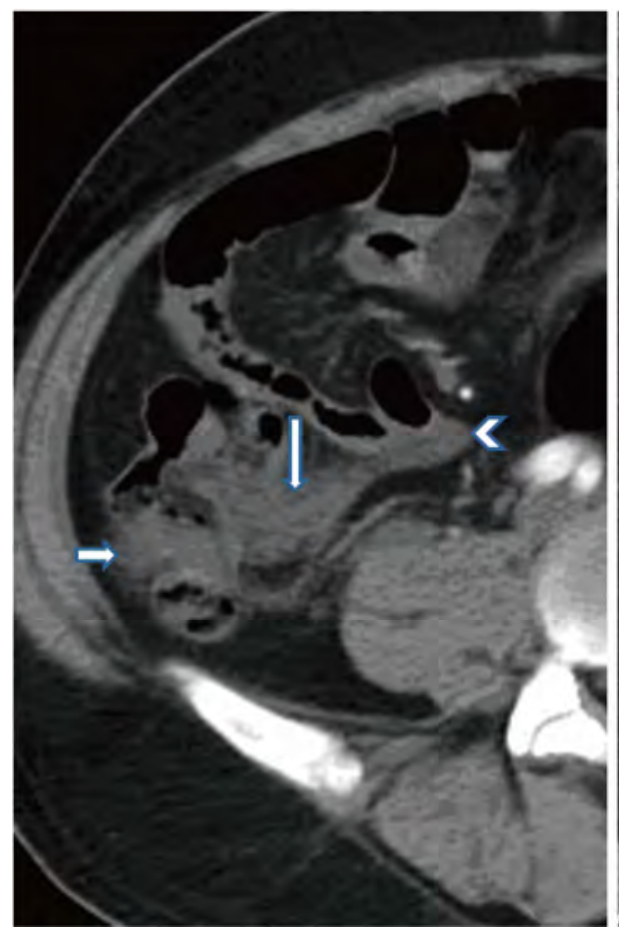

(a)

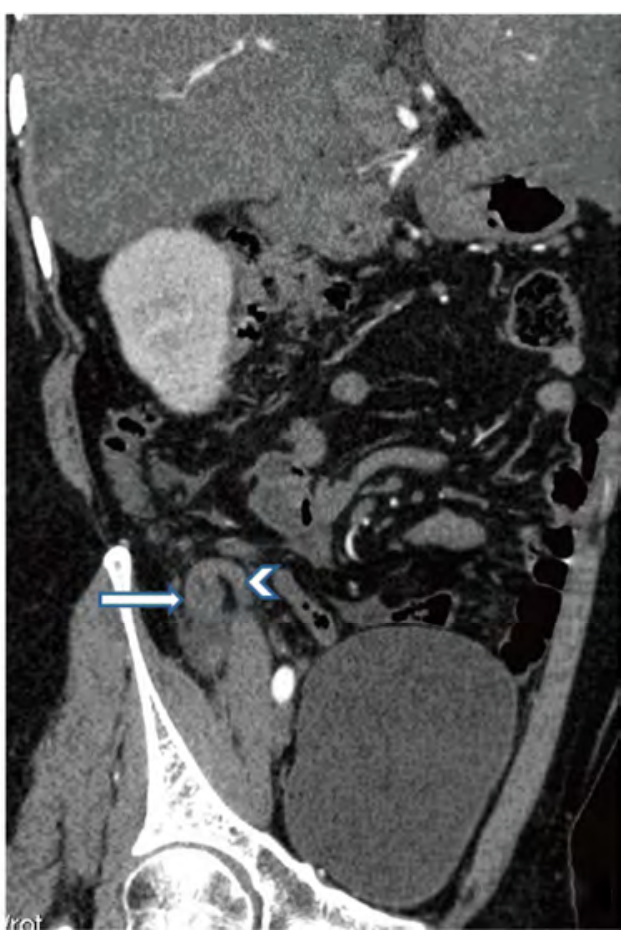

(b)

Figure 7. Axial nonenhanced (a) and Saggital oblique images shows thickening at its origin $(10 \mathrm{~mm})$. Body and tip of appendix (arrow head) is normal. Minimal ascites noted in right iliac fossa. (a, small arrow)
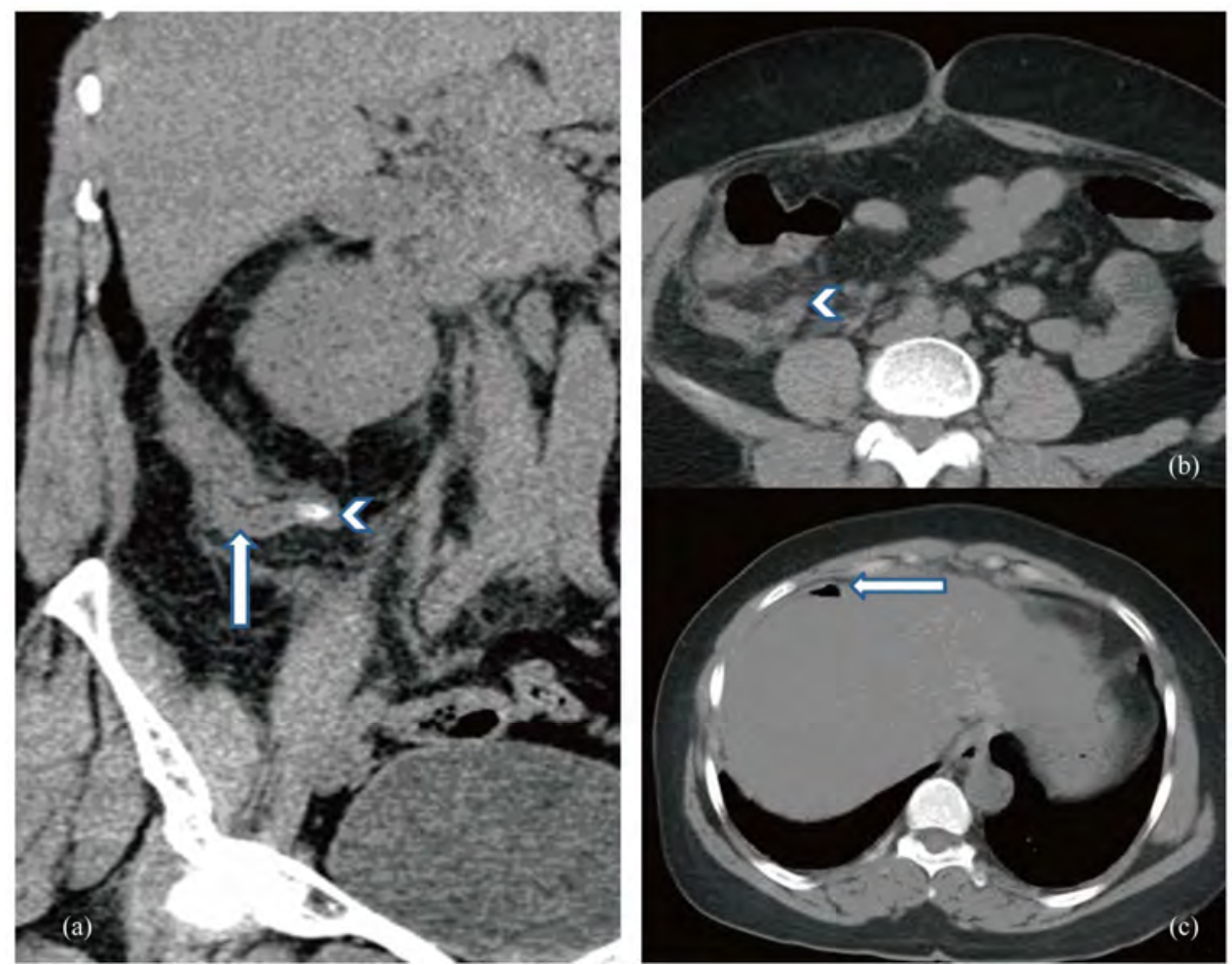

Figure 8. (a) Nonenhanced coronal Computed Tomography shows thickened tip (arrow head) with appendicolith within and adjacent fat stranding. Base of appendix (arrow) is normal. (b) Non enhanced axial Computed Tomography shows appendicolith (arrowhead) noted at the tip with surrounding mesenteric stranding. (c) Axial non enhanced Computed Tomography shows small pneumoperitoneum (arrow) in right sub diaphragmatic space. 


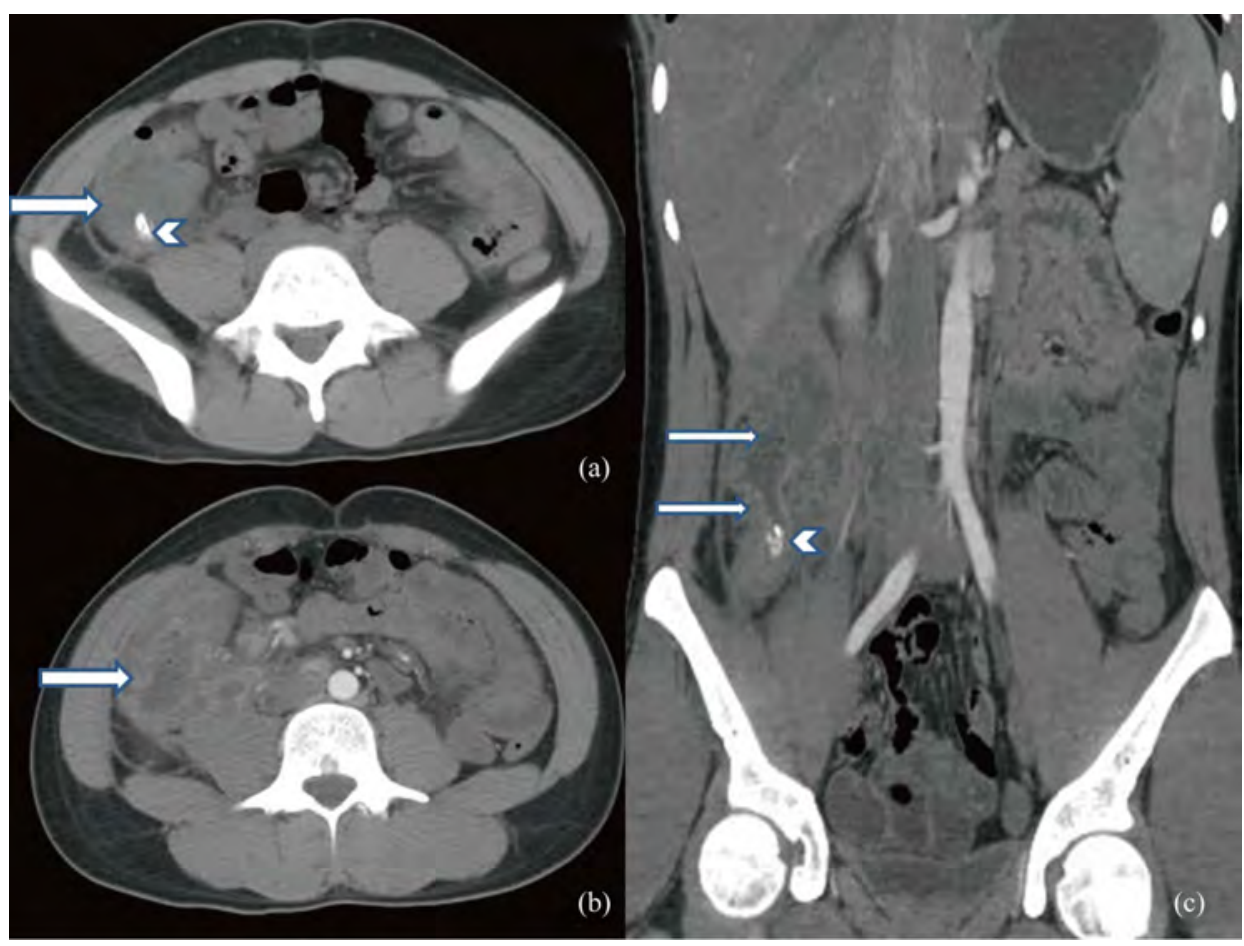

Figure 9. Axial nonenhanced (a) and enhanced (b) axial Computed Tomography shows complex heterogeneous mass in the right iliac fossa (arrow). Wall enhancement noted on contrast administration (b arrow). Large 14 mm appendicolith (arrow head) within the mass. (c) Right iliac fossa shows heterogeneous mass (arrow) fluid filled appendix (arrow) with appendicolith (arrowhead) within the lumen.

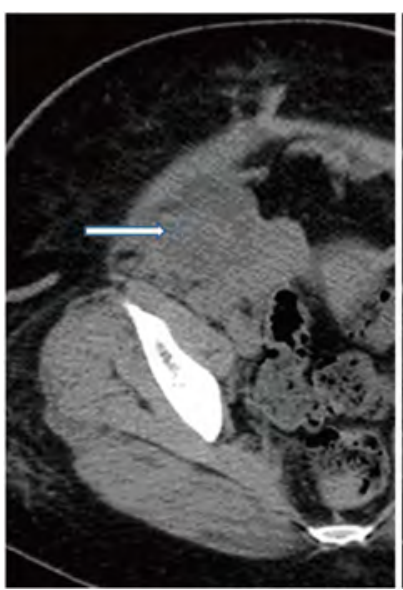

(a)

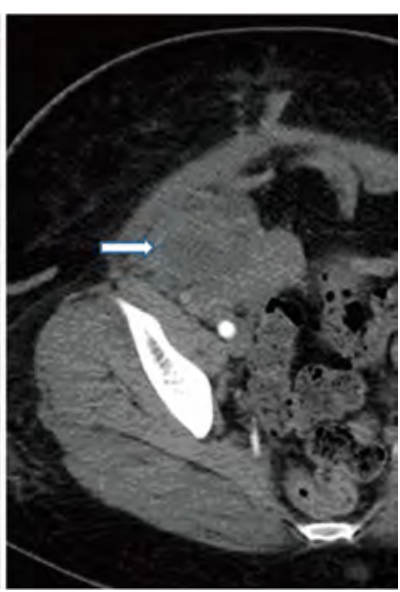

(b)

Figure 10. Nonenhanced (a) and enhanced (b) axial Computed Tomography shows ill-defined heterogeneous mass (a, arrow) in right iliac fossa. On contrast injection, wall enhancement (b, arrow) seen with necrotic areas within.

and Computed Tomography taken showed perforated appendix with adjacent air pockets(Figures 11(a) and 11(b)).

\subsection{Case XII Mucocele of Appendix}

Patient presented with mild lower abdominal pain for 1

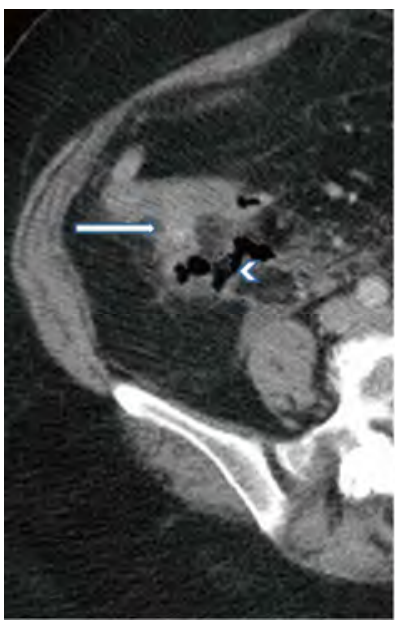

(a)

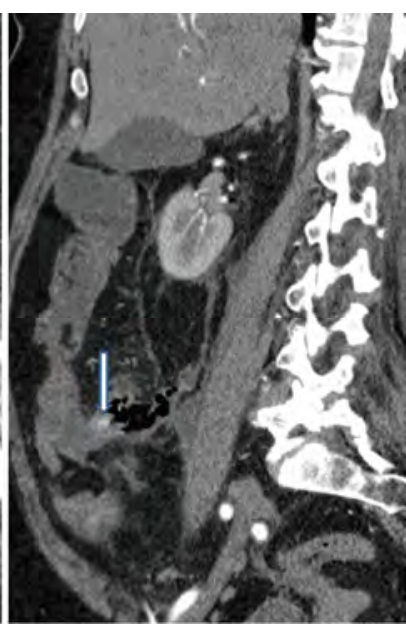

(b)
Figure 11. Axial nonenhanced (a) and enhanced coronal (b) Computed Tomography shows thickened appendix (arrow) at its take off from caecum with surrounding inflammed mesentery. Small Faecolith noted at the base. Focal gas pockets (arrowhead) noted adjacent to the appendix.

week and Computed Tomographic images showed distended edematous fluid filled appendix with appendicolith and air pockets within (Figures 12(a) and 12(b)). 


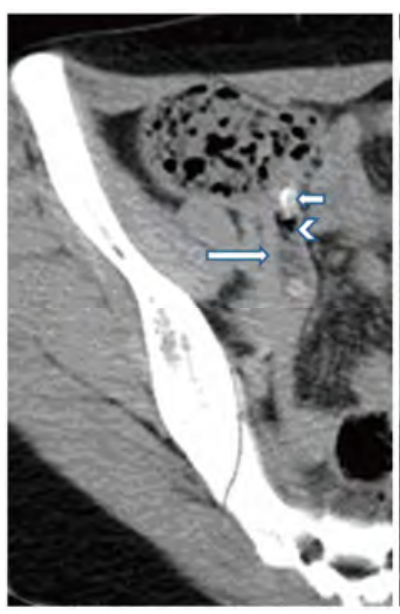

(a)

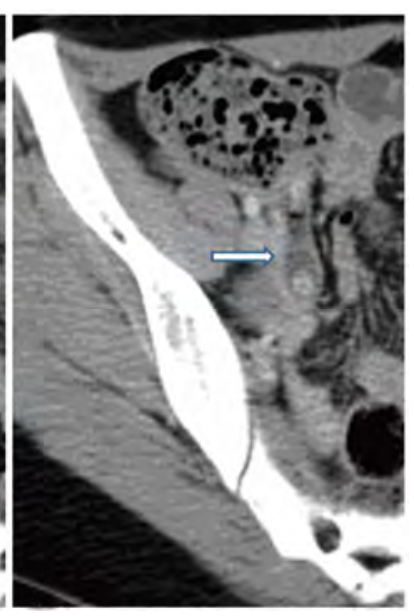

(b)
Figure 12. Nonenhanced (a) and enhanced (b) axial Computed tomography shows enlarged, edematous $(10 \mathrm{~mm})$ fluid filled appendix(arrow) with appendicolith (small arrow) in the base and few air pockets (a, arrowhead) seen within. Enhancement of appendicular wall (b, arrow) in Contrast Enhanced Computer Tomography.

\section{Pathophysiology}

The pathophysiology of appendicitis is the constellation of processes that leads to the development of acute appendicitis from a normal appendix. Appendicitis results due to obstruction of the appendiceal lumen. The main source of obstruction include lymphoid hyperplasia due to infection in the gastrointestinal tract and occasionally by inflammatory bowel disease (Crohn's disease and ulcerative colitis), feces, parasites, or growths that clog the appendiceal lumen and trauma to the abdomen

Understanding the pathophysiology helps to explain all the signs and symptoms and the complications seen in appendicitis. In all the cases, independent of etiology, the main thrust of events is increase in the pressure within the lumen followed by continuous secretion of fluids and mucus and the stagnation of this material leading to compromised blood supply and becoming very vulnerable to invasion by bacteria found in the gut normally.

The pathophysiology [7] involved in inflammation of appendix is as follows:

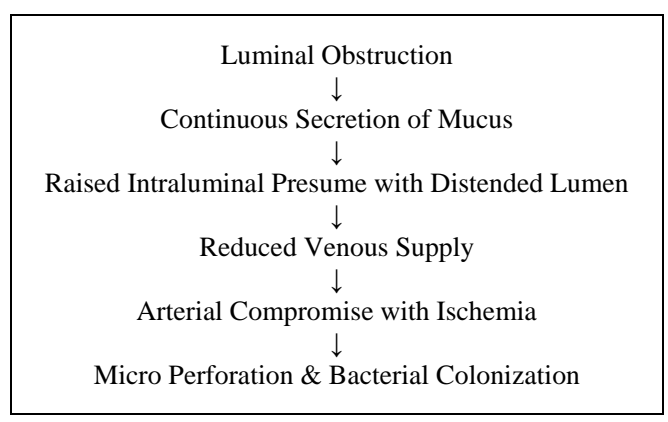

\section{Discussion}

Appendicitis is an acute inflammation of the vermiform appendix, typically resulting in abdominal pain, anorexia, and abdominal tenderness

Computed Tomography Criteria for Diagnosing Acute Appendicitis: Computed Tomography is a highly accurate and effective cross-sectional imaging technique for diagnosing and staging acute appendicitis. Abdominal and pelvic Computed Tomography scanning with or without oral \& intravenous contrast can be done, depending upon radiologist preference. However targeted Computed Tomography technique for evaluation of appendix is done in all the cases strongly suspicious of appendicular pathology. Computed Tomography scanning has the advantage of direct visualization of the appendix, periappendiceal region and other intra-abdominal structures.

There are primary as well as secondary signs for diagnosing appendicular pathologies on cross sectional imaging.

\section{Primary signs}

\begin{tabular}{|c|}
\hline Enlarged Unopacified Appendix \\
\hline Abnormal Thickened Appendix \\
\hline Abnormal Enhancement on CECT \\
\hline Periappendicular Fat Stranding [8] \\
\hline
\end{tabular}

Secondary signs

\begin{tabular}{|c|}
\hline Mesenteric Adenopathy \\
\hline Appendicolith \\
\hline Caecal Bar Sign—Focal Caecal Wall Thickening [9-11]. \\
\hline $\begin{array}{c}\text { Arrow Head Sign—Arrowhead Shaped Collection of } \\
\text { Contrast in Upper Part of Caecum near the Orifice of } \\
\text { Appendix [9-11]. }\end{array}$ \\
\hline Para Colic Gutter Fluid \\
\hline Diffuse Caecal Wall Thickening \\
\hline Abscess/Extra Luminal Air \\
\hline
\end{tabular}

The inflamed appendix usually measures $7-15 \mathrm{~mm}$ in diameter. Circumferential and symmetric wall thickening is always present and is best demonstrated on images obtained with intravenous contrast material enhancement. Homogenous enhancement of thickened wall is seen.

Other important findings include focal cecal apical thickening and the arrowhead sign (cecal contrast material funnels symmetrically at the cecal apex to the point of appendiceal occlusion). This secondary finding may 
help to establish the diagnosis in equivocal cases.

Enlargement of the appendix with associated fluid and loculated air within the lumen is seen in Gangrenous appendicitis.

Perforated appendicitis is usually accompanied by pericecal phlegmon or abscess formation. Other findings include extraluminal air, marked ileocecal thickening, localized lymphadenopathy, peritonitis, and small-bowel obstruction.

\section{Conclusions}

Computed Tomography can show a normal appendix as well as various other ways appendicitis can appear [12]. It is the accurate modality for the diagnosis of acute appendicitis, especially in patients with equivocal presentation. Radiologist must be aware of various clinical and radiological features of these presentations which aid in faster diagnosis and optimize treatment of these patients.

\section{References}

[1] D. Choi, H. Park, Y. R. Lee, S. H. Kook, S. K. Kim, H. J. Kwag, et al., "The Most Useful Findings for Diagnosing Acute Appendicitis on Contrastenhancedhelical CT," Acta Radiologica, Vol. 44, No. 6, 2003, pp. 547-582.

[2] A. N. Chalazonitis, I. Tzovara, E. Sammouti, N. Ptohis, E. Sotiropoulou, E. Protoppapa, et al., "CT in Appendicitis," Diagnostic and Interventional Radiology, Vol. 14, No. 1, 2008, pp. 19-25.

[3] T. Miki, S. Ogata and M. Uto, "Enhanced Multidetectorrow Computed Tomography (MDCT) in the Diagnosis of Acute Appendicitis and Its Severity,” Radiation Medicine, Vol. 23, 2005, pp. 242-255.

[4] A. J. Malone, "Unenhanced CT in the Evaluation of the Acute Abdomen: The Community Hospital Experience,"
Seminars in Ultrasound CT and MRI, Vol. 20, No. 2, 1999, pp. 68-76. doi:10.1016/S0887-2171(99)90038-0

[5] A. A. Ghiatas, S. Chopra and K. N. Chintapalli, "Computed Tomography of the Normal Appendix and Acute Appendicitis,” A. N. Chalazonitis, Ed., Diagnostic and Interventional Radiology, Vol. 11, 2005, pp. 45-50.

[6] K. R. Curtin, S. W. Fitzgerald, A. A. Nemcek, F. L. Hoff and R. L. Vogelzang, "Computed Tomography Diagnosis of Acute Appendicitis: Imaging Findings," American Journal of Roentgenology, Vol. 164, 1995, pp. 905-909.

[7] J. M. Pereira, et al., "Disproportionate Fat Stranding: A Helpful CT Sign in Patients with Acute Abdominal Pain," Radiographics, Vol. 24, May 2004, pp. 703-715. doi:10.1148/rg.243035084

[8] K. E. Applegate, C. J. Sivit, M. T. Myers and B. Pschesang, "Using Helical CT to Diagnosis Acute Appendicitis in Children: Spectrum of Findings," American Journal of Roentgenology, Vol. 176, No. 2, 2001, pp. 501505.

[9] E. J. Balthazar, B. A. Birnbaum, J. Yee, A. J. Megibow, J. Roshkow and C. Gray, "Acute Appendicitis: CT and US Correlation in 100 Patients,” Radiology, Vol. 190, 1994, pp. 31-33.

[10] P. M. Rao, J. T. Rhea and R. A. Novelline, "Sensitivity and Specificity of the Individual CT Signs of Appendicitis: Experience with 200 Helical Appendiceal CT Examinations," Journal of Computer Assisted Tomography, Vol. 21, No. 5, 1997, pp. 686-692. doi:10.1097/00004728-199709000-00002

[11] P. M. Rao, J. T. Rhea, R. A. Novelline, et al., "Helical CT Technique for the Diagnosis of Appendicitis: Prospective Evaluation of a Focused Appendix CT Examination," Radiology, Vol. 202, 1997, pp. 139-144.

[12] A. A. Ghiatas, S. Chopra and K. N. Chintapalli, "Computed Tomography of the Normal Appendix and Acute Appendicitis,” European Radiology, Vol. 7, 1997, pp. 1043-1047. doi:10.1007/s003300050249 